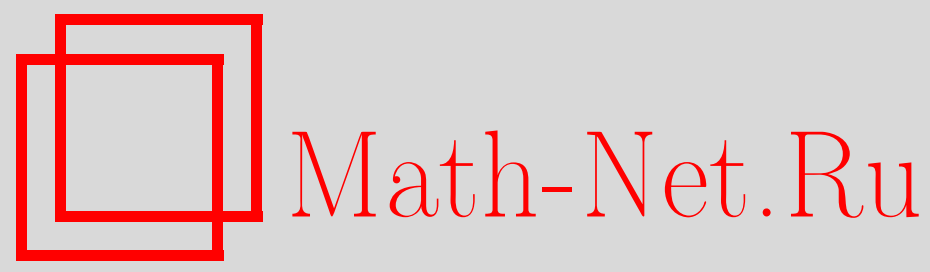

T. А. Береговая, А. М. Себельдин, Определяемость вполне разложимых абелевых групп без кручения группами гомоморфизмов, Матем. заметки, 2003, том 73, выпуск 5, 643648

DOI: https://doi.org/10.4213/mzm212

Использование Общероссийского математического портала Math-Net.Ru подразумевает, что вы прочитали и согласны с пользовательским соглашением http://www.mathnet.ru/rus/agreement

Параметры загрузки:

IP: 54.92 .164 .108

26 апреля 2023 г., 13:59:09

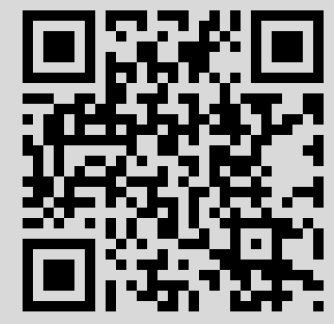




\title{
ОПРЕДЕЛЯЕМОСТЬ ВПОЛНЕ РАЗЛОЖИМЫХ АБЕЛЕВЫХ ГРУПП БЕЗ КРУЧЕНИЯ ГРУППАМИ ГОМОМОРФИЗМОВ
}

\author{
Т. А. Береговая, А. М. Себельдин
}

Пусть $C$ - абелева группа. Абелева группа $A$ из некоторого класса $\mathscr{X}$ абелевых групп $C H$-определяется в классе $\mathscr{X}$, если для всякой группы $B \in \mathscr{X}$ из изоморфизма $\operatorname{Hom}(C, A) \cong \operatorname{Hom}(C, B)$ следует изоморфизм $A \cong B$. Если каждая группа из $\mathscr{X}$ ${ }_{C} H$-определяется в $\mathscr{X}$, то класс $\mathscr{X}$ назьвается $C H$-классом. В статье исследуются условия, при которых класс вполне разложимых абелевых групп без кручения является ${ }_{C} H$-классом, где $C$ - вполне разложимая абелева группа без кручения.

Библиография: 8 названий.

Известная проблема 34 [1] была решена отрицательно в работах П. Хилла [2] и А. М. Себельдина [3], а именно, не существует такой абелевой группы $C$, что для любых групा $A, B$ из $\operatorname{Hom}(A, C) \cong \operatorname{Hom}(B, C)$ вытекает $A \cong B$. Кроме того, в [3] найдены вполне разложимые неизоморфные абелевы групшы $A$ и $B$ такие, что $\operatorname{Hom}(A, C) \cong \operatorname{Hom}(B, C)$ для любой абелевой группы $C$. В настоящей работе рассматривается обратная задача (см. также [4]).

Пусть $C$ - абелева группа. Класс $\mathscr{X}$ абелевых групп назовем $C H$-классом, если для любых групп $A, B \in \mathscr{X}$ из $\operatorname{Hom}(C, A) \cong \operatorname{Hom}(C, B)$ следует $A \cong B$.

В работе получены необходимые и достаточные условия, которым должна удовлетворять вполне разложимая абелева группа без кручения $C$, чтобы заданный подкласс класса $\Im_{c d}$ вполне разложимых абелевых групा без кручения был ${ }_{C} H$-классом.

Все групшы, рассматриваемые в работе, абелевы без кручения. Введем следуюшие обозначения: $\operatorname{rg}(A)$ - ранг группы $A, \tau(A)$ - тип абелевой групшы $A$ без кручения ранга $1, \Omega$-множество всех различных типов абелевых групп без кручения ранга $1, \Omega(A)$ множество всех различных типов прямых слагаемых ранга 1 вполне разложимой абелевой групшы $A$ без кручения, $\Omega_{0}(A)$ - множество всех типов из $\Omega(A)$, характеристики которых не содержат символов $\infty$.

Пусть

$$
A=\bigoplus_{i \in I} A_{i}, \quad B=\bigoplus_{j \in J} B_{j}, \quad C=\bigoplus_{k \in K} C_{k},
$$

где $\operatorname{rg}\left(A_{i}\right)=\operatorname{rg}\left(B_{j}\right)=\operatorname{rg}\left(C_{k}\right)=1,-$ вполне разложимые абелевы группы без кручения, имеющие следующие канонические разложения:

$$
A=\bigoplus_{\tau \in \Omega(A)} \bigoplus_{i \in I(\tau)} A_{i}, \quad B=\bigoplus_{\tau \in \Omega(B)} \bigoplus_{j \in J(\tau)} B_{j}, \quad C=\bigoplus_{\tau \in \Omega(C)} \bigoplus_{k \in K(\tau)} C_{k}
$$


где $I(\tau)=\left\{i \in I \mid \tau\left(A_{i}\right)=\tau\right\}, J(\tau)=\left\{j \in J \mid \tau\left(B_{j}\right)=\tau\right\}, K(\tau)=\left\{k \in K \mid \tau\left(C_{k}\right)=\right.$ $\tau\}$. Через $\left(\ldots, h_{p}^{\tau}, \ldots\right)$ обозначим характеристику, принадлежащую типу $\tau ; P_{\infty}(\tau)-$ множество тех простых чисел $p$, для которых $h_{p}^{\tau}=\infty$.

Tеорема 1. Пусть $C \in \Im_{c d}$. Класс $\Im_{c d}$ является ${ }_{C} H$-классом тогда и только тогда, когда группа $C$ удовлетворяет следующим условиям:

1) С имеет прямое слагаемое, изоморфное $\mathbf{Z}$;

2) $\Omega(C)$ содержит только идемпотентные типь (см. [5]);

3) $C$ имеет конечный ранг.

ДокАЗАТЕЛЬСтво. Достаточность. По условию $C=\bigoplus_{k=1}^{n} C_{k}$, где $\operatorname{rg}\left(C_{k}\right)=1$, $C_{1} \cong \mathbf{Z}$ и $\Omega(C)$ содержит только идемпотентные типы. Пусть $A, B \in \Im_{c d}$ такие, что $\operatorname{Hom}(C, A) \cong \operatorname{Hom}(C, B)$. Учитывая, что $\operatorname{rg}(C)<\aleph_{0}$, согласно [6] имеем

$$
A \oplus\left(\bigoplus_{k=2}^{n} \bigoplus_{i \in I} \operatorname{Hom}\left(C_{k}, A_{i}\right)\right) \cong B \oplus\left(\bigoplus_{k=2}^{n} \bigoplus_{j \in J} \operatorname{Hom}\left(C_{k}, B_{j}\right)\right) .
$$

Пусть $\tau_{0} \in \Omega(A)$. Так как $\tau(\mathbf{Z}) \in \Omega(C)$, то $\Omega(A) \subset \Omega(\operatorname{Hom}(C, A))$, откуда $\tau_{0} \in$ $\Omega(\operatorname{Hom}(C, A))$. Следовательно, $\tau_{0} \in \Omega(\operatorname{Hom}(C, B))$. Но поскольку все типы $\tau\left(C_{k}\right)$ идемпотентны, имеем $\operatorname{Hom}\left(C_{k}, B_{j}\right) \cong B_{j}$ или $\operatorname{Hom}\left(C_{k}, B_{j}\right)=0$. Таким образом, $\Omega(\operatorname{Hom}(C$, $B)) \subset \Omega(B)$ и $\tau_{0} \in \Omega(B)$, т.е. $\Omega(A) \subset \Omega(B)$. Обратное включение доказывается аналогично, откуда $\Omega(A)=\Omega(B)$.

Пусть $\left|T\left(\tau^{\prime}\right)\right|-$ мощность множества групп ранга 1 типа $\tau^{\prime}$ в разложении группы $\operatorname{Hom}(C, A)$. Тогда $\left|T\left(\tau^{\prime}\right)\right|=m^{\prime}\left|I\left(\tau^{\prime}\right)\right|$, где $m^{\prime} \neq 0$ - число групп $C_{k} \quad(k \in K)$, типы которых $\tau\left(C_{k}\right) \leqslant \tau^{\prime}$. С другой стороны, $\left|T\left(\tau^{\prime}\right)\right|=m^{\prime}\left|J\left(\tau^{\prime}\right)\right|$, откуда, учитьвая конечность $m^{\prime},\left|I\left(\tau^{\prime}\right)\right|=\left|J\left(\tau^{\prime}\right)\right|$. Следовательно, $A \cong B$.

Необходимость. Докажем сначала необходимость третьего условия. Предположим противное: $C=\bigoplus_{k \in K} C_{k}$, где $|K| \geqslant \aleph_{0}$.

Положим $A=\mathbf{Q}, B=\mathbf{Q} \oplus \mathbf{Q}$. Тогда

$$
\begin{gathered}
\operatorname{Hom}(C, A)=\operatorname{Hom}\left(\bigoplus_{k \in K} C_{k}, \mathbf{Q}\right) \cong \prod_{k \in K} \operatorname{Hom}\left(C_{k}, \mathbf{Q}\right) \cong \prod_{|K|} \mathbf{Q}, \\
\operatorname{Hom}(C, B)=\operatorname{Hom}\left(\bigoplus_{k \in K} C_{k}, \mathbf{Q} \oplus \mathbf{Q}\right) \cong \prod_{|K|}(\mathbf{Q} \oplus \mathbf{Q}) \\
\cong \prod_{|K|} \mathbf{Q} \oplus \prod_{|K|} \mathbf{Q} \cong \prod_{|K|} \mathbf{Q} .
\end{gathered}
$$

Таким образом, $\operatorname{Hom}(C, A) \cong \operatorname{Hom}(C, B)$, но $A \not B$.

Предположим теперь, что в разложении групшы $C$ нет прямых слагаемых, изоморфных группе Z. Тогда найдутся неизоморфные группы $A, B \in \Im_{c d}$ такие, что $\operatorname{Hom}(C, A) \cong$ $\operatorname{Hom}(C, B)$. Действительно, рассмотрим два случая.

1) $\Omega_{0}(C)=\varnothing$. Тогда возьмем группы $A$ и $B$ ранга 1 , типы которых содержат характеристики $(0,0,0, \ldots)$ и $(1,1,1, \ldots)$, соответственно. Очевидно, что $\operatorname{Hom}(C, A)=\operatorname{Hom}(C$, $B)=0$, но $A \supsetneqq B$.

2) $\Omega_{0}(C) \neq \varnothing$. Пусть $\tau_{l}$ - один из минимальных типов в $\Omega_{0}(C)$. Поскольку $\tau_{l} \neq$ $\tau(\mathbf{Z})$, во множестве $\Omega$ найдутся типы $\tau^{\prime}$ и $\tau^{\prime \prime}, \tau^{\prime} \neq \tau^{\prime \prime}$, такие, что $\tau^{\prime}<\tau_{l}$ и $\tau^{\prime \prime}<\tau_{l}$. 
Тогда в качестве $A$ и $B$ возьмем групшы ранга 1 типов $\tau^{\prime}$ и $\tau^{\prime \prime}$ соответственно. Ясно, что $\operatorname{Hom}(C, A)=\operatorname{Hom}(C, B)=0$ и $A \supsetneqq B$. Полученное противоречие доказывает необходимость первого условия.

Докажем необходимость второго условия теоремы. Предположим $C=C^{\prime} \oplus C^{\prime \prime}$, где $\tau(\mathbf{Z}) \in \Omega\left(C^{\prime}\right)$, все типы из $\Omega\left(C^{\prime}\right)$ идемпотентны, $C^{\prime \prime} \neq 0$ и все типы из $\Omega\left(C^{\prime \prime}\right)$ неидемпотентны и, наконец, $\operatorname{rg}(C)<\aleph_{0}$. Обозначим через $\tau^{0}$ идемпотентньй тип, соответствующий типу $\tau$, т.е., если $\mathbf{Q}(\tau)$ - группа ранга 1 типа $\tau$, то $\tau^{0}=\tau(\operatorname{Hom}(\mathbf{Q}(\tau), \mathbf{Q}(\tau)))$.

Положим

$$
\begin{aligned}
& A=A^{\prime} \oplus A^{\prime \prime}=\left(\bigoplus_{\tau \in \Omega\left(C^{\prime \prime}\right)} \bigoplus_{\alpha} \mathbf{Q}\left(\tau^{0}\right)\right) \oplus\left(\bigoplus_{\tau \in \Omega\left(C^{\prime \prime}\right)} \bigoplus_{\gamma} \mathbf{Q}(\tau)\right), \\
& B=B^{\prime} \oplus B^{\prime \prime}=\left(\bigoplus_{\tau \in \Omega\left(C^{\prime \prime}\right)} \bigoplus_{\beta} \mathbf{Q}\left(\tau^{0}\right)\right) \oplus\left(\bigoplus_{\tau \in \Omega\left(C^{\prime \prime}\right)} \bigoplus_{\gamma} \mathbf{Q}(\tau)\right),
\end{aligned}
$$

где $\alpha, \beta, \gamma$ - бесконечные кардиналы такие, что $\alpha<\beta<\gamma$. Учитьвая, что $\tau(\mathbf{Z}) \in \Omega\left(C^{\prime}\right)$, имеем

$$
\begin{aligned}
\operatorname{Hom}(C, A) & \cong \operatorname{Hom}\left(C^{\prime}, A\right) \oplus \operatorname{Hom}\left(C^{\prime \prime}, A\right) \\
& \cong A \oplus \operatorname{Hom}\left(C^{\prime \prime}, A^{\prime}\right) \oplus \operatorname{Hom}\left(C^{\prime \prime}, A^{\prime \prime}\right) \\
& \cong A^{\prime} \oplus A^{\prime \prime} \oplus \operatorname{Hom}\left(C^{\prime \prime}, A^{\prime}\right) \oplus \operatorname{Hom}\left(C^{\prime \prime}, A^{\prime \prime}\right) .
\end{aligned}
$$

Заметим, что в $\operatorname{Hom}\left(C^{\prime \prime}, A^{\prime \prime}\right)$ содержится прямое слагаемое, изоморфное $A^{\prime}$, и прямое слагаемое, изоморфное $\operatorname{Hom}\left(C^{\prime \prime}, A^{\prime}\right)$, поэтому

$$
A^{\prime} \oplus \operatorname{Hom}\left(C^{\prime \prime}, A^{\prime}\right) \oplus \operatorname{Hom}\left(C^{\prime \prime}, A^{\prime \prime}\right) \cong \operatorname{Hom}\left(C^{\prime \prime}, A^{\prime \prime}\right) .
$$

Таким образом,

$$
\operatorname{Hom}(C, A) \cong A^{\prime \prime} \oplus \operatorname{Hom}\left(C^{\prime \prime}, A^{\prime \prime}\right)
$$

Аналогично имеем

$$
\operatorname{Hom}(C, B) \cong B^{\prime \prime} \oplus \operatorname{Hom}\left(C^{\prime \prime}, B^{\prime \prime}\right) .
$$

Учитьвая, что $A^{\prime \prime} \cong B^{\prime \prime}$, получаем $\operatorname{Hom}(C, A) \cong \operatorname{Hom}(C, B)$ и $A \nsubseteq B$. Теорема доказана.

Заметим, что в классе вполне разложимых абелевых групп без кручения имеются подклассы, для которых соответственно условия 1,2 и 3 теоремы 1 не являются необходимыми. Как будет показано ниже, такими подклассами являются, например, следующие классы:

$-\Im_{c d}^{a d}$ почти делимых вполне разложимых групп (т.е. для $G \in \Im_{c d}^{a d}$ выполнено $p G=G$ почти для всех простых $p$ );

- $\Im_{c d}^{*}$ вполне разложимых групп $A$, где $\Omega(A)$ содержит только несравнимые типы;

- $\Im_{1}$ групп ранга 1.

ПредЛОЖенИЕ 1. Пусть $C \in \Im_{c d}$. Класc $\Im_{c d}^{a d}$ почти делимых вполне разложимых абелевых групп без кручения является ${ }_{C} H$-классом тогда и только тогда, когда группа С удовлетворяет следующим условиям:

1) $\Omega_{0}(C) \neq \varnothing$

2) $C$ имеет конечный ранг. 
ДоКАЗАТЕЛЬСТВО. Достаточность. Пусть условия 1 и 2 вьполнены. Рассмотрим $A, B \in \Im_{c d}^{a d}$ такие, что $\operatorname{Hom}(C, A) \cong \operatorname{Hom}(C, B)$. Пусть $C=C^{\prime} \oplus C^{\prime \prime}$, где

$$
C^{\prime}=\bigoplus_{\tau \in \Omega_{0}(C)} \bigoplus_{k \in K(\tau)} C_{k}, \quad C^{\prime} \neq 0, \quad C^{\prime \prime}=\bigoplus_{\tau \in \Omega(C) \backslash \Omega_{0}(C)} \bigoplus_{k \in K(\tau)} C_{k}
$$

Тогда

$$
\operatorname{Hom}(C, A) \cong \operatorname{Hom}\left(C^{\prime}, A\right) \oplus \operatorname{Hom}\left(C^{\prime \prime}, A\right) \cong \bigoplus_{\operatorname{rg}\left(C^{\prime}\right)} A \oplus \operatorname{Hom}\left(C^{\prime \prime}, A\right)
$$

Следовательно, $\Omega(A) \subset \Omega(\operatorname{Hom}(C, A))$. Как и при доказательстве достаточности в теореме 1 можно показать, что $\Omega(A)=\Omega(B)$ и $|I(\tau)|=|J(\tau)|$. Следовательно, $A \cong B$.

Необходимость второго условия доказана в теореме 1 , так как построенные там группы $A$ и $B$ принадлежат классу $\Im_{c d}^{a d}$. Докажем необходимость первого условия. Пусть, от противного, для любого типа $\tau$ из $\Omega(C) P_{\infty}(\tau) \neq \varnothing$. Построим множество $P^{\prime}$, выбрав по одному $p$ из каждого множества $P_{\infty}(\tau)$. Тогда в качестве группы А возьмем группу ранга 1 , тип которой содержит характеристику $\left(\ldots, h_{p}, \ldots\right)$, где $h_{p} \neq \infty$ при $p \in P^{\prime} ; h_{p}=\infty$ при $p \notin P^{\prime}$. Положим $B=A \oplus A$. Очевидно, что $A \not B$, но $\operatorname{Hom}(C, A)=\operatorname{Hom}(C, B)=0$. Противоречие. Предложение 1 доказано.

ПРЕДЛОЖЕНИЕ 2. Пусть $C \in \Im_{c d}$. Класс $\Im_{1}$ является $C H$-классом тогда $и$ только тогда, когда группа С удовлетворяет одному из следующ, их условий:

1) С содержит прямое слагаемое, изоморфное $\mathbf{Z}$;

2) $\tau(\mathbf{Z}) \notin \Omega(C)$, и для любого типа $\tau^{\prime} \in \Omega$, где $\tau^{\prime} \neq \tau(\mathbf{Z})$, найдется тип $\tau \in \Omega(C)$ такой, что $\tau \leqslant \tau^{\prime}$.

ДокАЗАтЕЛЬСтво. Достаточность. Пусть $C$ удовлетворяет первому условию теоремы, т.е. $\tau(\mathbf{Z}) \in \Omega(C)$. Пусть $\operatorname{Hom}(C, A) \cong \operatorname{Hom}(C, B)$. Так как $A, B \in \Im_{1}$, ясно, что $\operatorname{Hom}(C, A)$ редуцированная тогда и только тогда, когда $A$, а значит, и $B$ редуцированные. В этом случае $\operatorname{Hom}(C, A)$ - векторная группа [5] и, следовательно [7], $\operatorname{Hom}(C, A)$ и $\operatorname{Hom}(C, B)$ обладают изоморфными разложениями

$$
\prod_{k \in K} \operatorname{Hom}\left(C_{k}, A\right) \cong \prod_{k \in K} \operatorname{Hom}\left(C_{k}, B\right)
$$

в частности, $\Omega(\operatorname{Hom}(C, A))=\Omega(\operatorname{Hom}(C, B))$. Так как $\tau(\mathbf{Z}) \in \Omega(C)$, то $\tau(A)=\tau(\operatorname{Hom}(\mathbf{Z}$, $A))$ и $\tau(B)$ принадлежат $\Omega(\operatorname{Hom}(C, A))=\Omega(\operatorname{Hom}(C, B))$. Далее, найдется индекс $k \in$ $K$ такой, что $\tau(A)=\tau\left(\operatorname{Hom}\left(C_{k}, B\right)\right)$. Из [8] следует, что $\tau(A) \leqslant \tau(B)$. Аналогично получаем, что $\tau(B) \leqslant \tau(A)$. Следовательно, $\tau(B)=\tau(A)$.

Пусть теперь групша $C=\bigoplus_{k \in K} C_{k}, \operatorname{rg}\left(C_{k}\right)=1$, удовлетворяет второму условию теоремы. Предположим, что существуют такие неизоморфные группы $A$ и $B$ ранга 1 , что $\operatorname{Hom}(C, A) \cong \operatorname{Hom}(C, B)$. Как и в первом случае, достаточно рассмотреть случай, когда $A$ и $B$ редуцированные. Тогда [5]

$$
\prod_{k \in K} \operatorname{Hom}\left(C_{k}, A\right) \cong \prod_{k \in K} \operatorname{Hom}\left(C_{k}, B\right)
$$


и $\Omega(\operatorname{Hom}(C, A))=\Omega(\operatorname{Hom}(C, B))($ см. [7]). Зафиксируем в типе $\tau(A)$ групшы $A$ характеристику $\left(\ldots, \alpha_{p}, \ldots\right)$, в типе $\tau(B)$ групшы $B$ характеристику $\left(\ldots, \beta_{p}, \ldots\right)$. Положим

$$
P_{1}=\left\{p \in P \mid \beta_{p}>\alpha_{p}\right\}, \quad P_{2}=\left\{p \in P \mid \alpha_{p}>\beta_{p}\right\} .
$$

Пусть, например, $\left|P_{1}\right| \geqslant\left|P_{2}\right|$. Возможны два случая.

a) $\left|P_{1}\right|=\aleph_{0}$. Разобьем множество $P_{1}=P_{1}^{\prime} \cup P_{1}^{\prime \prime}$ так, чтобы $\left|P_{1}^{\prime}\right|=\left|P_{1}^{\prime \prime}\right|=\aleph_{0}$. Построим характеристику $\left(\ldots, \gamma_{p}, \ldots\right)$ следуюшим образом: $\gamma_{p}=1$, если $p \in P_{1}^{\prime}$, и $\gamma_{p}=0$, если $p \notin P_{1}^{\prime}$, и пусть $\tau$ - тип, определяемьй этой характеристикой. В разложении группы $C$ существует такое прямое слагаемое $C_{1}$ ранга 1 , что $\tau\left(C_{1}\right)=\tau_{1}, \tau_{1} \leqslant \tau$ и $\tau_{1} \neq \tau(\mathbf{Z})$. Согласно [8] $\operatorname{Hom}\left(C_{1}, B\right) \neq 0$ и $\tau\left(\operatorname{Hom}\left(C_{1}, B\right)\right)=\tau(B)-\tau_{1}=\bar{\tau}$. Далее, тип $\bar{\tau}$ либо несравним с $\tau(A)$, либо больше его, так как $\bar{\tau}$ содержит характеристику $\left(\ldots, \bar{\beta}_{p}, \ldots\right)$, где для всех $p \in P_{1}^{\prime \prime} \bar{\beta}_{p}=\beta_{p}>\alpha_{p}$. С другой стороны, для любого прямого слагаемого $C_{k}$ имеем $\tau\left(\operatorname{Hom}\left(C_{k}, A\right)\right) \leqslant \tau(A)$ или $\operatorname{Hom}\left(C_{k}, A\right)=0$. Следовательно, в разложении группы $\operatorname{Hom}(C, A)$ нет групшы, изоморфной группе $\operatorname{Hom}\left(C_{1}, B\right)$, откуда $\operatorname{Hom}(C, A) \supsetneqq \operatorname{Hom}(C, B)$, что противоречит условию.

б) $\left|P_{1}\right|<\aleph_{0}$. Тогда найдется $q \in P_{1}$ с условием $\beta_{q}=\infty$. Далее, в $\Omega(C)$ существует тип $\tau_{0}=\tau\left(Q^{(q)}\right.$ ) (здесь $\tau\left(Q^{(q)}\right)$ - подгруппа рациональных чисел, знаменатели которых - степени $q)$. Имеем $\tau\left(\operatorname{Hom}\left(C_{0}, B\right)\right)=\tau(B)-\tau_{0}=\bar{\tau}_{0}$. Заметим, что тип $\bar{\tau}_{0}$ больше типа $\tau(A)$, либо несравним с ним, так как $\bar{\tau}_{0}$ содержит характеристику $\left(\ldots, \bar{\gamma}_{p}, \ldots\right)$, где $\bar{\gamma}_{q}=\beta_{q}=\infty$. Тогда, как и в случае а), получаем, что $\operatorname{Hom}(C, A) \not \operatorname{Hom}(C, B)$.

Необходимость. Пусть для типа $\tau^{\prime}$ из $\Omega, \tau^{\prime} \neq \tau(\mathbf{Z})$, в $\Omega(C)$ не найдется меньшего или равного $\tau^{\prime}$ типа. Рассмотрим две неизоморфные групшы $A$ и $B$ ранга 1 такие, что $\tau(A)=\tau(\mathbf{Z}), \tau(B)=\tau^{\prime}$. Тогда $\operatorname{Hom}(C, A)=\operatorname{Hom}(C, B)=0$. Полученное противоречие доказьвает необходимость условий теоремы. Предложение 2 доказано.

СледСТВИЕ 1. Пусть $C \in \Im_{c d}$. Eсли $\Omega_{0}(C)$ - конечное множество (например, если $\left.\operatorname{rg}(C)<\aleph_{0}\right)$, то класс $\Im_{1}$ является $C H$-классом в том и только в том случае, когда группа $C$ содержит прямое слагаемое, изоморфное группе $\mathbf{Z}$.

ПРЕДЛОЖЕНИЕ 3. Пусть $C \in \Im_{c d}$. Класс $\Im_{c d}^{*}$ является $C$ Н-классом тогда и только тогда, когда группа С удовлетворяет следуюшим условиям:

1) С содержит прямое слагаемое, изоморфное $\mathbf{Z}$;

2) $C$ имеет конечный ранг.

ДоКАЗАТЕЛЬСТво. Достаточность. Пусть

$$
\begin{gathered}
A=\bigoplus_{i \in I} A_{i}=\bigoplus_{\tau \in \Omega(A)} \bigoplus_{i \in I(\tau)} A_{i}, \quad \operatorname{rg}\left(A_{i}\right)=1, \\
B=\bigoplus_{j \in J} B_{j}=\bigoplus_{\tau \in \Omega(B)} \bigoplus_{j \in J(\tau)} B_{j}, \quad \operatorname{rg}\left(B_{j}\right)=1, \\
C=\bigoplus_{k=1}^{n} C_{k}, \quad C_{1} \cong \mathbf{Z}, \quad \operatorname{rg}\left(C_{k}\right)=1
\end{gathered}
$$

- фиксированные разложения груп $A, B$ и $C$ соответственно.

Учитывая, что $\operatorname{rg}(C)<\aleph_{0}$, согласно [6] из

$$
\operatorname{Hom}(C, A) \cong \operatorname{Hom}(C, B)
$$


следует

$$
A \oplus\left(\bigoplus_{k=2}^{n} \bigoplus_{i \in I} \operatorname{Hom}\left(C_{k}, A_{i}\right)\right) \cong B \oplus\left(\bigoplus_{k=2}^{n} \bigoplus_{j \in J} \operatorname{Hom}\left(C_{k}, B_{j}\right)\right) .
$$

Тогда для любой групшы $A_{i}$ найдется группа $\operatorname{Hom}\left(C_{k}, B_{j}\right)$ такая, что $A_{i} \cong \operatorname{Hom}\left(C_{k}, B_{j}\right)$, откуда $\tau\left(B_{j}\right) \geqslant \tau\left(A_{i}\right)$. Аналогично $B_{j} \cong \operatorname{Hom}\left(C_{t}, A_{l}\right)$ для некоторой групшы $A_{l}$ и $\tau\left(B_{j}\right) \leqslant \tau\left(A_{l}\right), t \in\{1, \ldots, n\}$. Следовательно, $\tau\left(A_{i}\right) \leqslant \tau\left(B_{j}\right) \leqslant \tau\left(A_{l}\right)$, а так как все типы из $\Omega(A)$ несравнимы, то $A_{i} \cong B_{j}$.

Аналогично для любой групшы $B_{j}$ найдется группа $A_{i}$ такая, что $B_{j} \cong A_{i}$. Следовательно, $\Omega(A)=\Omega(B)$.

Пусть $\tau \in \Omega(A)$. Тогда $\tau\left(\operatorname{Hom}\left(C_{k}, A_{i}\right)\right)=\tau$ для некоторых $i \in I(\tau), k \in\{1,2, \ldots, n\}$, откуда $\tau\left(A_{i}\right)=\tau$, поскольку различные типы из $\Omega(A)$ попарно несравнимы. Далее, имеем $|T(\tau)|=m(\tau)|I(\tau)|$, где $|T(\tau)|$ - мошность множества групп ранга 1 типа $\tau$ из $\operatorname{Hom}(C, A), m(\tau)$ - число груп $C_{k}$, типы которых идемпотентны относительно типа $\tau$ (т.е. $\tau-\tau\left(C_{k}\right)=\tau$ ). С другой стороны, $|T(\tau)|=m(\tau)|J(\tau)|$. Следовательно, $A \cong B$.

Необходимость второго условия доказана в теореме 1 , так как построенные там группы $A$ и $B$ принадлежат классу $\Im_{c d}^{*}$.

Докажем необходимость первого условия. Пусть в разложении групшы $C$ нет слагаемых, изоморфных групе $\mathbf{Z}$. Тогда положим $A \cong \mathbf{Z}, B \cong \mathbf{Z} \oplus \mathbf{Z}$. Очевидно, что $A, B \in \Im_{c d}^{*}$ и $\operatorname{Hom}(C, A)=\operatorname{Hom}(C, B)=0$, но $A \nsubseteq B$. Предложение 3 доказано.

\section{СПИСОК ЦИТИРОВАННОЙ ЛИТЕРАТУРЫ}

[1] Fuchs L. Resent Results and Problems on Abelian groups // Topics in Abelian groups. Chicago, 1963. P. 9-40.

[2] Hill P. Two problems of L. Fuchs Concerning Tor and Hom // J. Algebra. 1971. V. 19. №3. P. 379-383.

[3] Себельдин А. М. О группах гомоморфизмов абелевых групп без кручения // Группы и модули. 1976. С. 70-77.

[4] Антонова Н. Ю. Определяемость абелевых групп и модулями группами и модулями гомоморфизмов. Дис. ... канд. физ.-матем. наук. М.: МПГУ, 1994.

[5] Фукс Л. Бесконечные абелевы группы. Т. 1, 2. М.: Мир, 1977.

[6] Sebeldin A. M. Isomorphisme naturel des groupes des homomorphismes des groupes abeliens // Ann. de L'IPGANG. Conakry. Ser. A. 1982. V. 7. P. 155-158.

[7] Мишина А. П. О прямых слагаемых полных сумм абелевых групп без кручения ранга 1 // Сиб. матем. ж. 1962. № 3. С. 244-249.

[8] Себельдин А. М. Группы гомоморфизмов вполне разложимых абелевых групп без кручения // Изв. ВУЗов. Матем. 1973. № 7. С. 77-84. 